\title{
Revisión taxonómica y geográfica de Bryconamericus peruanus (Teleostei, Characidae)
}

\section{Taxonomic and geographic revision of Bryconamericus peruanus (Teleostei, Characidae)}

\author{
César Román-Valencia ${ }^{1 *}$, María Dolly García ${ }^{2}$ y Hernán Ortega ${ }^{3}$ \\ ${ }^{1}$ Universidad del Quindio, Laboratorio de Ictiología, A. A. 2639, Armenia, Colombia. \\ ${ }^{2}$ Universidad del Quindio, Grupo de Investigación y Asesoría en Estadística, Armenia, Colombia. \\ ${ }^{3}$ Departamento de Ictiología-Museo de Historia Natural, Universidad Mayor de San Marcos, Apartado 11434, Lima 14, Perú. \\ *Correspondencia: ceroman@uniquindio.edu.co
}

\begin{abstract}
Resumen. Se efectúa un análisis taxonómico y geográfico de Bryconamericus peruanus y se compara con las especies de este género presentes en las vertientes del Pacífico sur en Colombia, Ecuador y Perú. Bryconamericus peruanus restringe su distribución al sistema hidrográfico del Pacífico en Perú y se plantea que algunas formas del Pacífico y Amazonas de Ecuador son especies por describir. Los caracteres asociados al dimorfismo sexual, como ganchos en todas las aletas con radios excepto la aleta caudal, son útiles en la diferenciación de B. peruanus y Bryconamericus sp.1 con respecto a B. brevirostris, B. dahli, B. guaytarae, B. miraensis, Bryconamericus sp. 2 y Bryconamericus sp. 3. De acuerdo con la forma (de la mandíbula superior, longitud aleta dorsal-hipurales y longitud pedúnculo caudal), el análisis de componentes principales distingue 2 grupos de especies: I) B. peruanus, B. brevirostris, B. guaytarae, B. miraensis y Bryconamericus sp. 3 y II) B. dahli, Bryconamericus sp. 1 y Bryconamericus sp. 2.
\end{abstract}

Palabras clave: taxonomía, B. peruanus, poblaciones, distribución, pez tropical.

\begin{abstract}
Bryconamericus peruanus were analyzed and compared with species belonging to the genus Bryconamericus that occurs in the Pacific basin from Colombia, Ecuador and Peru. Distribution range of B. peruanus is restricted to Peruvian drainages and the populations from the Pacific basin and those located at the Amazons in Ecuador are regarded new species. The presence of hooks on all the fins, except on the caudal fin, was the most reliable character to separate populations of B. peruanus and Bryconamericus $\mathrm{sp} .1$ from B. brevirostris, B. dahli, B. guaytarae, B. miraensis, Bryconamericus sp. 2 and Bryconamericus sp. 3. According to the form upper jaw, dorsal fin-hypurals distance and caudal peduncle length from the principal component analysis, 2 groups are distinguished: I) B. peruanus, B. brevirostris, B.guaytarae, B. miraensis y Bryconamericus sp. 3, and II) B. dahli, Bryconamericus sp. 1 and Bryconamericus sp. 2.
\end{abstract}

Key words: taxonomy, B. peruanus, populations, distribution, tropical fish.

\section{Introducción}

Las especies de Bryconamericus han sido poco estudiadas en algunos países; por ejemplo, en Ecuador, Perú y Bolivia (en ambas cuencas del Pacífico y Amazonas) (Román-Valencia, 2003a; 2005; Román-Valencia et al., 2008a; b; 2009a; b; c; d; 2010). A pesar de que existen trabajos sobre la taxonomía y sistemática de Bryconamericus, e incluso se han descrito nuevas especies de este género en el Pacífico de Ecuador y Colombia (Böhlke, 1958; Román-Valencia, 2003a; 2005; Román-Valencia y Vanegas-Ríos, 2009), no hay estudios concluyentes sobre el patrón de distribución geográfica de Bryconamericus para la vertiente del Pacífico de Ecuador y Perú, lo que se

Recibido: 12 enero 2009; aceptado: 4 febrero 2011 ve reflejado en la existencia de registros raros y aparentemente erróneos de algunas de las especies del género; es el caso de B. scleroparius restringida a Panamá y Costa Rica (Román-Valencia, 2002) que ha sido registrada para Ecuador.

Actualmente se reconoce la existencia de 5 especies de Bryconamericus, que se distribuyen en la vertiente sur del Pacífico de Colombia y occidente de Ecuador: $B$. brevirostris, B. dahli, B. peruanus, B. guaytarae y $B$. miraensis (Román-Valencia, 2003a; 2005; Román-Valencia et al., 2008b).

Bryconamericus peruanus, descrita por Müller y Troschel (1845), se recolectó por primera vez en la cuenca hidrográfica del Pacífico en Perú alrededor de Lima, pero su distribución se amplía a varias localidades del occidente de Ecuador y Colombia (Eigenmann, 1927; Román-Valencia, 2005; Román-Valencia et al., 2008b). 
Esta especie también se ha registrado en cuencas hidrográficas del Atlántico y alto Amazonas (Böhlke, 1958; Ortega y Vari, 1986).

Se ha planteado que posiblemente $B$. peruanus $\mathrm{y}$ B. caucanus que se distribuyen en la cuenca del río Cauca, Sinú y Ranchería en Colombia (Eigenmann, 1927; Román-Valencia et al., 2009a; b) son sinónimos (Tortonese, 1941; 1942); sin embargo, no se presentan evidencias que sustenten esta idea.

Desde esta perspectiva, el objetivo del trabajo es analizar la variación morfológica y geográfica de $B$. peruanus, las especies de Bryconamericus de la vertiente sur del Pacífico de Colombia, en Ecuador y Perú.

\section{Materiales y métodos}

Se examinaron 180 ejemplares de Bryconamericus peruanus ( 2 sintipos, y 178 topotipos), 25 individuos de $B$. guaytarae, 10 de B. miraensis, 116 de B. brevirostris y 74 de $B$. dahli; 75 ejemplares de $B$. peruanus del Pacífico y alto Amazonas de Ecuador se les incluyó como Bryconamericus sp. 1, y 30 como Bryconamericus sp. 2. Además, se examinaron 48 ejemplares de la cuenca del río Mira en el Pacífico de Colombia a los cuales se les denominóBryconamericus sp. $3(\mathrm{n}=48)$. En total se inspeccionaron 558 ejemplares de Bryconamericus del Pacífico en Colombia, Ecuador y Perú. Es importante señalar que también se tomaron en cuenta algunos ejemplares de las cuencas más cercanas, como la de los ríos Piano $(n=2)$, Bitumaco $(n=2)$ y Amarillo $(n=4)$ en Ecuador.
El material antes relacionado se encuentra depositado en los museos y colecciones anotadas a continuación. En los acrónimos se siguió a Sabaj-Pérez (2010): Laboratorio de Ictiología de la Universidad del Quindío, Armenia, Colombia (IUQ), Academy of Natural Sciences, Phyladelphia, Pennsylvania, USA (ANSP), British Museum, Londres, Inglaterra (BMNH), California Academy of Sciences, USA (CAS), Universidad Nacional de Colombia, Museo de Historia Natural, Unidad de Ictiología, Bogotá (ICNMNH), Escuela Politécnica Nacional de Quito, Museo de Zoología, Ecuador (MEPN), Museo de Historia Natural de la Universidad Nacional Mayor de San Marcos, Lima, Perú (MUSM), National Museum of Natural History, Smithsonian Institution, Washington, D.C. (USNM), Universität Humboldt, Museum für Naturkunde, Berlín (ZMB).

Variables. Las medidas (Cuadros 1 y 2) se tomaron con calibrador digital hasta décimas de milímetro, todas fueron obtenidas punto a punto; además, se obtuvieron medidas de los sintipos de $B$. peruanus por medio del programa Scion Image Beta 4.02 (Scion Corporation, disponible en: www.scioncorp.com). Los conteos de escamas y radios se realizaron a través de un estereoscopio sobre el lado izquierdo de los ejemplares, excepto cuando éstos estaban deteriorados en dicho lado. Los conteos merísticos y variables morfométricas utilizados siguen a Fink y Weitzman (1974), con las modificaciones de RománValencia (2003b). Se contó el número de vértebras, radios procurrentes en la aleta caudal y pterigióforos proximales de la aleta anal y los ganchos presentes en las aletas anal,

Cuadro 1. Datos morfométricos y merísticos de las especies de Bryconamericus del Pacífico de Colombia y Ecuador. Longitud estándar y total en mm. Promedios entre paréntesis

\begin{tabular}{|c|c|c|c|c|}
\hline & $\begin{array}{l}\text { B. brevirostris } \\
(\mathrm{n}=117)\end{array}$ & $\begin{array}{l}\text { B. dahli } \\
(\mathrm{n}=74)\end{array}$ & $\begin{array}{l}\text { B. miraensis } \\
(\mathrm{n}=10)\end{array}$ & $\begin{array}{l}\text { B. guaytarae } \\
(\mathrm{n}=25)\end{array}$ \\
\hline Longitud estándar & $32.9-65.6(43.1)$ & $31.1-75.3(53.7)$ & $67.8-90.1(75.7)$ & $26.6-76.1(47.1)$ \\
\hline Longitud total & $14.9-79.4(54.0)$ & $41.4-91.8(68.1)$ & $84.1-109.5$ (89.9) & $34.3-94.1(59.2)$ \\
\hline \multicolumn{5}{|l|}{ Porcentaje de la longitud estándar } \\
\hline Profundidad del cuerpo & $21.5-54.6(35.8)$ & $28.9-49.1(36.1)$ & $36.2-47.0(42.2)$ & $45.1-51.2(47.9)$ \\
\hline Longitud hocico-aleta dorsal & $35.3-71.4(47.6)$ & $17.5-65.9(47.8)$ & $50.1-63.8(59.0)$ & $58.7-67.9(62.9)$ \\
\hline Longitud hocico-aleta pectoral & $16.1-29.5(20.7)$ & $28.8-39.3(21.3)$ & $18.4-25.0(22.5)$ & $23.3-29.6(26.2)$ \\
\hline Longitud hocico-aleta pélvica & $30.7-60.1(40.5)$ & $24.8-59.9(42.1)$ & $44.8-54.6(51.4)$ & $47.8-56.0(51.7)$ \\
\hline Longitud hocico-aleta anal & $16.8-39.4(24.2)$ & $15.2-42.1(28.9)$ & $26.6-40.7(31.9)$ & $28.5-34.7(32.3)$ \\
\hline Longitud aleta dorsal-hipurales & $21.7-44.8(29.6)$ & $18.2-48.5(32.6)$ & $31.9-45.8(39.6)$ & $36.3-43.2(40.2)$ \\
\hline Longitud aleta dorsal-aleta anal & $5.2-11.2(7.2)$ & 4.7-14.1 (8.9) & $10.5-13.6(11.9)$ & $10.3-13.2(11.5)$ \\
\hline Longitud aleta dorsal-aletas pectorales & $2.9-9.8(5.2)$ & $2.9-12.8(6.5)$ & $6.9-17.7(12.2)$ & $7.7-12.1(9.3)$ \\
\hline Longitud aleta dorsal & $9.9-26.0(16.7)$ & $8.9-29.2(17.8)$ & $16.8-22.1(19.4)$ & $17.3-25.0(21.5)$ \\
\hline Longitud aletas pectorales & $7.7-19.4(11.3)$ & $5.7-16.5(11.6)$ & $12.4-16.7(14.5)$ & $12.1-17.1(14.7)$ \\
\hline Longitud aletas pélvicas & $10.2-28.3(18.2)$ & $11.4-28.5(20.1)$ & $16.8-24.8(21.3)$ & $14.3-29.9(24.2)$ \\
\hline Longitud aleta anal & $10.4-20.1(13.9)$ & $5.6-21.7(14.3)$ & $13.1-19.2(15.8)$ & $14.6-21.7(17.9)$ \\
\hline Profundidad del pedunculo caudal & $14.6-26.1(18.4)$ & $4.4-29.7(20.7)$ & $17.4-20.7(19.7)$ & $21.1-26.2(23.4)$ \\
\hline
\end{tabular}


Cuadro 1. Continúa

\begin{tabular}{|c|c|c|c|c|}
\hline & $\begin{array}{l}\text { B. brevirostris } \\
(\mathrm{n}=117)\end{array}$ & $\begin{array}{l}\text { B. dahli } \\
(\mathrm{n}=74)\end{array}$ & $\begin{array}{l}\text { B. miraensis } \\
(\mathrm{n}=10)\end{array}$ & $\begin{array}{l}\text { B. guaytarae } \\
(\mathrm{n}=25)\end{array}$ \\
\hline Longitud del pedunculo caudal & $15.5-37.6(23.2)$ & $15.6-41.9(28.5)$ & $25.4-38.3(30.8)$ & $27.4-33.8(31.2)$ \\
\hline Longitud cabeza & $27.7-39.7(34.3)$ & $22.8-54.8(39.8)$ & $45.0-56.1(52.5)$ & $48.9-55.8(52.3)$ \\
\hline \multicolumn{5}{|l|}{ Porcentaje de la longitud de la cabeza } \\
\hline Longitud hocico & $14.3-31.62(20.4)$ & $4.2-24.3(9.2)$ & $12.9-16.9(14.7)$ & $14.1-19.22(17.1)$ \\
\hline Diámetro del ojo & 16.3-49.7 (36.9) & $2.3-13.9(5.4)$ & $8.6-12.5(10.3)$ & 8.4-13.9 (11.9) \\
\hline Longitud postorbital de la cabeza & $13.6-34.3(25.8)$ & $4.8-19.4(8.5)$ & $10.5-16.3(12.2)$ & $13.7-20.2(17.1)$ \\
\hline Longitud mandíbula superior & $16.6-33.4(23.2)$ & $0.7-7.5(2.8)$ & 9.8-13.1 (11.6) & $11.6-17.4(14.6)$ \\
\hline \multicolumn{5}{|l|}{ Merística } \\
\hline Escamas sobre la línea lateral & $38-50$ & $34-35$ & $39-43$ & $37-39$ \\
\hline Escamas entre la línea lateral y la aleta dorsal & $5-9$ & - & $5-7$ & $6-7$ \\
\hline Escamas entre la línea lateral y la aleta anal & $6-10$ & - & $4-6$ & $6-7$ \\
\hline Escamas entre la línea lateral y la aleta ventral & $6-9$ & - & $4-6$ & $6-7$ \\
\hline Escamas predorsales & $10-14$ & $12-13$ & $12-15$ & $10-13$ \\
\hline Radios de la aleta dorsal & ii,6 & - & ii, 7-9 & iii, 8 \\
\hline Radios de la aleta anal & ii, $10-12$ & ii, 29-31 & iv, 22-28 & iii, 23-24 \\
\hline Radios de la aleta ventral & iii, 8 & - & i-ii, 6-7 & ii,6 \\
\hline Radios de la aleta pectoral & iii-v, 33-40 & - & ii, $9-11$ & i, $10-12$ \\
\hline
\end{tabular}

Cuadro 2. Datos morfométricos y merísticos de lo registrado como Bryconamericus spp. y B. peruanus. Longitud estándar y total en $\mathrm{mm}$. Promedios entre paréntesis

\begin{tabular}{|c|c|c|c|c|}
\hline & $\begin{array}{l}\text { B. peruanus } \\
(\mathrm{n}=179)\end{array}$ & $\begin{array}{l}\text { Bryconamericus } \\
\quad \text { sp. } 1(\mathrm{n}=75)\end{array}$ & $\begin{array}{l}\text { Bryconamericus } \\
\quad \text { sp. } 2(\mathrm{n}=30)\end{array}$ & $\begin{array}{l}\text { Bryconamericus } \\
\text { sp. } 3(\mathrm{n}=48)\end{array}$ \\
\hline Longitud estándar & $29.5-89.3(59.0)$ & $21.1-92.3(56.7)$ & $37.4-72.9(56.4)$ & $22.6-96.6(54.5)$ \\
\hline Longitud total & $37.5-103(73.2)$ & $27.1-111.8(71.8)$ & $48.1-89.5(69.3)$ & $28.6-116.7(66.9)$ \\
\hline \multicolumn{5}{|l|}{ Porcentaje de la longitud estándar } \\
\hline Profundidad del cuerpo & $27.7-79.7(52.2)$ & $40.9-51.5(46.7)$ & $28.8-49.2(44.9)$ & $24.5-52.1(46.5)$ \\
\hline Longitud hocico-aleta dorsal & $16.1-107.1(68.5)$ & $57.7-69.1(63.2)$ & $50.9-68.3(62.2)$ & $42.6-69.2(62.2)$ \\
\hline Longitud hocico-aleta pectoral & $14.5-39.5(27.7)$ & $22.6-29.9(26.4)$ & $22.0-26.5(23.8)$ & $22.3-31.3(25.2)$ \\
\hline Longitud hocico-aleta pélvica & $28.8-89.0(59.0)$ & $51.6-57.9(54.8)$ & $45.8-57.9(53.7)$ & $31.6-56.4(52.7)$ \\
\hline Longitud hocico-aleta anal & $15.2-60.9(37.8)$ & $29.9-51.2(37.7)$ & $30.1-37.0(33.2)$ & $24.6-38.4(32.9)$ \\
\hline Longitud aleta dorsal-hipurales & $22.1-60.9(45.9)$ & $37.8-45.9(42.5)$ & $36.8-45.4(41.4)$ & $31.9-45.0(39.9)$ \\
\hline Longitud aleta dorsal-aleta anal & $6.0-22.4(14.0)$ & $9.7-14.6(12.3)$ & $10.5-13.7(11.9)$ & 5.6-19.9(11.7) \\
\hline Longitud aleta dorsal-aletas pectorales & $3.4-21.8(9.7)$ & $6.0-12.0(8.9)$ & $9.1-16.3(12.7)$ & $2.7-14.6(10.5)$ \\
\hline Longitud aleta dorsal & $9.2-34.3(22.6)$ & $20.1-27.3(22.9)$ & $16.9-21.6(19.7)$ & $11.6-23.4(20.2)$ \\
\hline Longitud aletas pectorales & $8.1-23.9(16.2)$ & $12.3-18.2(15.8)$ & $12.2-16.3(14.0)$ & $6.3-23.0(13.9)$ \\
\hline Longitud aletas pélvicas & $12.9-37.5(24.6)$ & $20.0-40.7(26.4)$ & $17.5-24.8(22.4)$ & $15.3-26.4(23.1)$ \\
\hline Longitud aleta anal & $9.7-26.3(17.6)$ & $11.1-22.2(18.3)$ & $9.2-20.4(14.8)$ & $9.4-33.3(16.7)$ \\
\hline Profundidad del pedúnculo caudal & $13.8-56.4(35.6)$ & $\begin{array}{l}20.8-29.6(24.9) \\
20.8-29.6(24.9)\end{array}$ & $21.5-24.6(23.1)$ & $19.2-28.8(24.7)$ \\
\hline Longitud del pedúnculo caudal & $13.6-35.5(24.3)$ & $29.0-42.6(36.82)$ & $30.4-39.1(34.4)$ & $26.6-44.2(33.1)$ \\
\hline Longitud cabeza & $31.8-45.4(39.6)$ & $48.3-54.8(50.9)$ & $42.9-51.2(47.1)$ & $43.5-53.1(49.9)$ \\
\hline \multicolumn{5}{|l|}{ Porcentaje de la longitud de la cabeza } \\
\hline Longitud hocico & $15.9-47.9(31.5)$ & $14.4-23.5(18.5)$ & $18.7-28.6(23.7)$ & $17.4-30.8(22.4)$ \\
\hline Diámetro del ojo & $27.7-52.3(39.7)$ & $8.2-20.3(11.3)$ & $8.5-14.0(11.9)$ & 9.2-15.8 (11.9) \\
\hline Longitud postorbital de la cabeza & $18.5-49.9(35.7)$ & $13.2-25.4(19.0)$ & $10.7-19.6(14.4)$ & $11.6-22.6(16.2)$ \\
\hline Longitud mandíbula superior & $19.7-61.7(38.2)$ & $12.9-16.8(14.6)$ & $3.9-7.4(5.9)$ & $3.8-16.4(7.1)$ \\
\hline \multicolumn{5}{|l|}{ Merística } \\
\hline Escamas sobre la línea lateral & $35-40$ & $37-39$ & $32-37$ & $34-36$ \\
\hline Escamas entre la línea lateral y la aleta dorsal & $5-7$ & 6-7 & 6-8 & $6-7$ \\
\hline Escamas entre la línea lateral y la aleta anal & $5-7$ & $5-7$ & $4-7$ & $5-7$ \\
\hline
\end{tabular}


Cuadro 2. Continúa

\begin{tabular}{|c|c|c|c|c|}
\hline & $\begin{array}{l}\text { B. peruanus } \\
(\mathrm{n}=179)\end{array}$ & $\begin{array}{l}\text { Bryconamericus } \\
\quad \text { sp. } 1(\mathrm{n}=75)\end{array}$ & $\begin{array}{l}\text { Bryconamericus } \\
\quad \text { sp. } 2(\mathrm{n}=30)\end{array}$ & $\begin{array}{l}\text { Bryconamericus } \\
\text { sp. } 3(\mathrm{n}=48)\end{array}$ \\
\hline Escamas entre la línea lateral y la aleta ventral & $5-7$ & $5-7$ & $5-6$ & $5-7$ \\
\hline Escamas predorsales & $10-13$ & $12-14$ & $12-14$ & $12-14$ \\
\hline Radios de la aleta dorsal & ii,6 & i-ii, 8-9 & ii,7 & iii, 8 \\
\hline Radios de la aleta anal & iii-iv, 23-28 & ii-iii, 27-29 & iii, $22-26$ & iii, 26-32 \\
\hline Radios de la aleta ventral & iii, $7-8$ & i-ii, 6-7 & ii,6-7 & ii,6 \\
\hline Radios de la aleta pectoral & i-ii, 9-11 & i, $10-12$ & ii,9-10 & i-ii, $9-12$ \\
\hline
\end{tabular}

pectoral, pélvicas dorsal y caudal de ejemplares maduros en todas las especies identificadas en este trabajo y las registradas para Centro y Suramérica (incluye a Colombia y Venezuela), sobre ejemplares clareados y teñidos (C y T) de acuerdo con las modificaciones al método descrito por Taylor y Van Dyke (1985) y Song y Parenti (1995). Los conteos de vértebras incluyen las 4 primeras del aparato de Weber y el centrum terminal. Se evaluaron los ganchos presentes en las aletas con radios de machos maduros en todas las especies identificadas en éste trabajo y las registradas para Centro y Suramérica (incluye Colombia y Venezuela).

Análisis estadístico. Para analizar la formación de grupos dentro de las especies de Bryconamericus del Pacífico de Colombia, Ecuador y Perú, se realizó un análisis de componentes principales (ACP) con los 23 caracteres morfométricos (Cuadros 1 y 2), y se aplicó la técnica de corrección a la talla (Burnaby, 1966). Las poblaciones de cada especie de Bryconamericus que se encontraron dentro del área de estudio fueron comparadas con las especies reconocidas como válidas.

Se realizó un análisis de varianza de un factor con la prueba de Kruskal-Wallis, junto a una comparación múltiple de Mann-Whitney utilizando los caracteres merísticos. En estos casos, se utilizaron los paquetes estadísticos SPSS 9.0, Statgraphics 5.0 (Statgraphics, 2005) y Past 1.81 (Paleontological Statistics) (Hammer et al., 2001).

Material examinado. Bryconamericus peruanus (Fig. 1): Todos de Perú: ZMB 3591 (2) cerca de Lima (sintipos). CAS 69032 (25) Lima, río Rimac. CAS 69002 (15) Lima, río Rimac cuenca Chosica. CAS 69029 (15), río Rimac, puente Piedra cerca de Lima. CAS 68994 (3), Lima, río Rimac cuenca Chosica, afluente río Santa Eulalia. CAS 69003 (5), Lima, río Rimac cuenca río Rimac. MUSM 334636 (3), Lima, río Chillón, Trapiche Km. 40 en la vía a Canta. MUSM 12539 (5), Lima, río Chillón, Puente Trapiche. MUSM 10270 (23), Lima, Cañete, Imperial R. Cañocte. MUSM 7332 (9), Piura, río Sitopiura. MUSM 35228 (39), Lambayeque, Chiclayo, R. Udima. MUSM (24), Lambayeque, Chiclayo, río Zaria a $224 \mathrm{~m}$ snm. MUSM (9), Lambayeque, Chiclayo, río Zaña. MUSM
35226 (1), Lambayeque, Chiclayo, embalse Oyotum a 191 m snm.MUSM 30418 (7), Ancash, Santa El Castillo. MUSM 6865 (15), Lima, Pantanos de villa canal abierto, estación hidrométrica.15706 (5). Ancash, Huari, Palca, río Puchka. 18642. (4), Cajamarca, Contumazos, río Jequetepequel Granada $\left(7^{\circ} 15^{\prime} 27.75^{\prime \prime} \mathrm{S}-7^{\circ} 38^{\prime} 48,6\right.$ O) 1336 m snm. MUSM 18038 (14), Cajamarca, Contumaza, río Jequetepeque Trasval ( $\left.7^{\circ} 13^{\prime} 27,5^{\prime \prime} \mathrm{S}-78^{\circ} 48^{\prime} 31,4^{\prime \prime} \mathrm{O}\right) 918$ m snm. MUSM 23123 (46), Piura, Tambogrande, Piura. MUSM 35238 (5), Lambayeque, Chiclayo, río Zaña. MUSM 0166 (18), Tumbes, río Tumbes. MUSM6255 (20), Tumbes, Zarumilla, Matapalo, río Zarumilla, quebrada Facial. B. brevirostris: Todos de Perú, departamento de Tumbes: MUSM 2582 (20), río Tumbes, cerca de La irrigación, Bocatoma. MUSM 5732 (7), San Jacinto en Bocatoma, río Tumbes. MUSM (6), río Tumbes, canal del río. MUSM 3393 (3), San Jacinto, canal de Bocatoma. MUSM (1), Zarumilla, Matapalo, río Zarumilla. MUSM 5765 (33), Zarumilla, La Panga, río Zarumilla. MUSM 5740 (8), Zarumilla, Matapalo, quebrada Faical. Todos de Ecuador, provincia de Guayas: CAS 72032 (1), río Peripa. CAS 68950 (1), río Guayas río Daule, Colimes. CAS 68948 (15), ríos Guayas-Chanchan (tributario río Chimbo, río Guayas), Naranjo. CAS 22749 (10), río Guayas, río Daule Colimes. CAS 68947 (20) río Daule, Colimes. CAS 68949 (21), río Provincia, río Guayas. CAS 68946 (50), río Guayas, río Daule, Colimes. MUSM (3), cuenca río Guayas, Cotimes, río Daule. B. dahli: Todos de Ecuador: MEPN 9-4063 (15), Esmeraldas, estero finca Kolynos a $2 \mathrm{~km}$ de la desembocadura en el río Cachari, río Bogotá. MEPN 19 (5), Guayas, río Minas, cruza cooperativa 23 noviembre a $9 \mathrm{~km}$ al sur de Naranjal. MEPN 3-4308 (10), Esmeraldas, río Matajita afluente del río Mataje media hora del centro comunal Mataje, vía hacia el río Mira y estero Pensamiento a $800 \mathrm{~m}$ aguas del sitio El Pan. MEPN 8-4074 (15), Esmeraldas, estero Pistolas media hora aguas abajo de Vargas Torres. B. guaytarae: Todos de Colombia: CAS 40844 (1 paratipo), Nariño, cuenca río Patia, río Guaitara en la boca con el río Patia. IUQ 364 (1), Nariño, cuenca del río Patia, río Nambi. IUQ 330 (23), Cauca, Patia, cuenca del río Patia, quebrada La Pulida en 


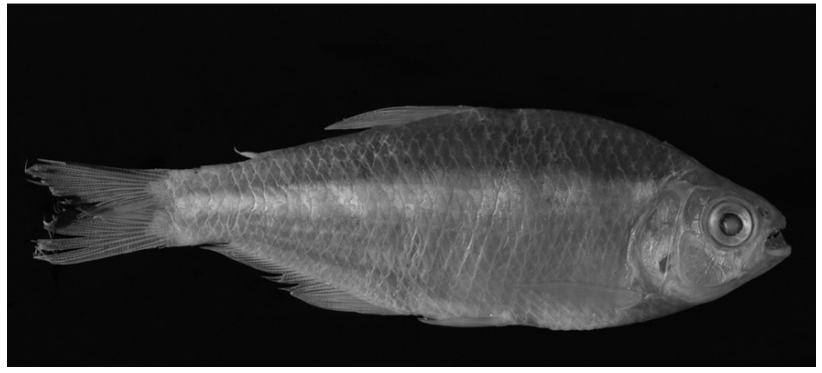

Figura 1. Bryconamericus peruanus. Sintipo: ZMB 3591: 70.8 mm LE, vertiente Pacífico, cerca de Lima, Perú.

su desembocadura, finca La Mica ( $2^{\circ} 09^{\prime} 26^{\prime \prime} \mathrm{N}, 77^{\circ} 24^{\prime}$ 00" O) 572 m. IUQ 333 (11), Cauca, Patia, vereda Angulo, río Patia en alrededores de Upesca $\left(2^{\circ} 02^{\prime} 40^{\prime \prime} \mathrm{N}, 77^{\circ} 06^{\prime}\right.$ 59” O) 752 m. ICNMNH 4882 (39), Nariño, cuenca del río Patia, río Nambi. ICNMNH 4883 (18), Nariño, cuenca del río Patia. IUQ 439 (7), Nariño, Patía entre Florida Baja y Patia, cuenca del río Patia, quebrada Palobobo $\left(2^{\circ} 4^{\prime}\right.$ 28” N, 77²' 43" O) 597 m. IUQ 438 (5), Cauca, Patia, cuenca del río Patia, quebrada el Zanjón $\left(2^{\circ} 4^{\prime} 32^{\prime}\right.$ N, $77^{\circ}$ 05' 00" O) 612 m. IUQ 481 (22) (se colectaron muertos), Nariño, Barbacoas, sistema del río Patia, cuenca del río Telembi, en bocas del río Yamunde en el río Telembi, 1 km debajo de Barbacoas (1'39'56" N, 78 9' 12" O) 61 m. B. miraensis: todos B. miraensis: Todos de Colombia: ANSP 71686 (Holotipo), Nariño, Ricaurte, cuenca del río Mira, en el río Guebo a 3900 pies de altura. ANSP 71687 (6 paratipos) colectados con el holotipo. Bryconamericus sp. 1: Todos de Ecuador: MEPN 13-378 (10), Esmeraldas, río Piripe a $300 \mathrm{~m}$ del puente Gualea a Porto. MEPN 16-3979 (15), Esmeraldas, estero Boca delonsole margen derecha del río Guayllabamba a $450 \mathrm{~m}$ Golondrina. MEPN 18- 4004 (5), Esmeraldas, estero Tatica a $2 \mathrm{~km}$ del recinto El Timbre. MEPN 20 (5), Guayas, estero Vicenso Santo canal río Areniclas. MEPN 23 (3), Oro, río Negro afluente del río Amarillo cerca de Portobello. MEPN 24 (10), Loja, río Macara aguas arriba de la ciudad de Macara. MEPN 26 (12), Oro, sitio Huertas en el sitio Jubones. Bryconamericus sp. 2: Ecuador: MEPN 42 (30), Zamora-Chinchipe, río Chicará alto afluente R. Zamora. Bryconamericus sp. 3: Todos de Colombia: ICNMNH 2352 (2), Nariño, Tumaco, río Mira cerca de Caunapi. IUQ 444 (9), Nariño, Tumaco; vertiente del Pacífico, afluente río Agua Clara, quebrada Iguapi en la vía Tumaco-Chimpí (1 $40^{\prime} 42^{\prime \prime} \mathrm{N}, 78^{\circ} 45^{\prime}$ 21 ” O). IUQ 445 (2), Nariño, Tumaco, río Mira en Candelillas. IUQ 275 (5), Nariño, Tumaco, río Mira cerca de la Y en la vía Tumaco-Caunapi. IUQ 442 (25), Nariño, Tumaco, vertiente del Pacífico, afluente del río Caunapi, quebrada Tangarial (1 $1^{\circ} 33^{\prime} 14^{\prime \prime} \mathrm{N}, 78^{\circ} 41^{\prime} 25^{\prime \prime}$ O) $30 \mathrm{~m}$. BMNH 6.16:169-172 (4) (determinación errónea: $B$. scleroparius). USNM 164060 (3), Ecuador, provincia de
Napo-Pastaza, río Pucuno tributario río Suno $\left(00^{\circ} 46^{\prime} \mathrm{S}\right.$, $\left.77^{\circ} 12^{\prime} \mathrm{O}\right)$.

\section{Resultados}

La forma de las especies de Bryconamericus del Pacífico de Colombia, Ecuador y Perú son muy similares, no obstante el análisis de componentes principales para la morfometría mostró 2 agrupaciones de acuerdo con la forma: el primero agrupa a B. brevirostris, B. guaytarae, B. peruanus, B. miraensis y Bryconamericus sp. 3 , y el segundo conformado por $B$. dahli, Bryconamericus sp. 1 y Bryconamericus sp. 2; las variables que mejor explican el patrón de variación obtenido son: longitud mandíbula superior, longitud pedúnculo caudal (sobre componente 1) y longitud aleta dorsal-hipurales (sobre componente 2) con $51.16 \%$ de la variación explicada (Cuadros 3 y 4, Fig. 2).

Los caracteres morfométricos mostraron que la forma de Bryconamericus peruanus para las cuencas hidrográficas de Perú difiere de lo registrado para el Pacífico de Ecuador (Bryconamericus sp. 1), Amazonas de Ecuador (Bryconamericus sp. 2) y Pacífico sur de Colombia (Bryconamericus sp. 3) y constituyen 3 especies por describir (Cuadro 2, Figs. 2 y 3). El análisis de componentes principales para los caracteres morfométricos mostró que las variables mejor representadas y correlacionadas son: longitud mandíbula superior (sobre componente 2), longitud aleta dorsal-hipurales y longitud pedúnculo caudal (sobre componente 1) con $62.70 \%$ de varianza (Cuadro 3 y 4, Fig. 3). El caracter sexual secundario como los ganchos presentes en todas las aletas con radios, excepto la caudal, distingue a $B$. peruanus y a Bryconamericus sp. 1 de $B$. brevirostris, $B$. dahli, B. guaytarae, B. miraensis, Bryconamericus sp. 2 y Bryconamericus sp. 3 que sólo presentan ganchos en los radios de las aletas anal y pélvica.

Los siguientes caracteres permiten distinguir a Bryconamericus miraensis de las demás especies de Bryconamericus del Pacífico de Colombia, Ecuador y Perú: número de escamas con poros en la línea lateral (39-42 vs. $<39, \mathrm{n}=441$ ) (Kruskal Wallis: $p<0.0001)$ y número total de radios de la aleta anal (26-28 vs. $>28$, excepto Bryconamericus sp. 2, $\mathrm{n}=441$ ) (Kruskal Wallis: $p<0.0001$ ) (Cuadros 1 y 2). De acuerdo con la prueba Kruskal-Wallis Bryconamericus sp. 2 se distingue de $B$. miraensis por el número de escamas con poros en la línea lateral (32-37 vs. <39). Bryconamericus sp. 2 se separa de $B$. dahli por el número de radios totales en la aleta anal $(25-29$ vs. $<31)$.

El número de vértebras, de radios procurrentes en la aleta caudal y pterigiòforos proximales de la aleta anal no distinguen las especies de Bryconamericus de la cuenca hidrográfica del Pacífico de Colombia, Ecuador y Perú. Sin 
Cuadro 3. Eigenvalor y porcentajes de variación del analisis de componentes principales de las especies de Bryconamericus del Pacífico de Colombia, Ecuador y Perú. CP: componente principal

\begin{tabular}{|c|c|c|c|c|c|}
\hline \multicolumn{4}{|c|}{ Bryconamericus en drenajes de Colombia, Ecuador y Perú } & \multicolumn{2}{|c|}{ Registrado Bryconamericus peruanus } \\
\hline$C P$ & Eigenvalor & $\%$ Varianza & $C P$ & Eigenvalor & $\%$ Varianza \\
\hline 1 & 0.245697 & 51.167 & 1 & 0.322619 & 62.704 \\
\hline 2 & 0.0741689 & 15.446 & 2 & 0.047988 & 9.327 \\
\hline 3 & 0.0308543 & 6.4255 & 3 & 0.0235045 & 4.5684 \\
\hline 4 & 0.0197735 & 4.1179 & 4 & 0.0203664 & 3.9584 \\
\hline 5 & 0.0153291 & 3.1923 & 5 & 0.0173196 & 3.3663 \\
\hline 6 & 0.0149311 & 3.1094 & 6 & 0.0147922 & 2.875 \\
\hline 7 & 0.0123213 & 2.566 & 7 & 0.0115036 & 2.2358 \\
\hline 8 & 0.0110915 & 2.3098 & 8 & 0.00886486 & 1.723 \\
\hline 9 & 0.0100176 & 2.0862 & 9 & 0.00793523 & 1.5423 \\
\hline 10 & 0.00883323 & 1.8395 & 10 & 0.00678513 & 1.3188 \\
\hline 11 & 0.00674506 & 1.4047 & 11 & 0.00608361 & 1.1824 \\
\hline 12 & 0.00594779 & 1.2386 & 12 & 0.00521496 & 1.0136 \\
\hline 13 & 0.00499448 & 1.0401 & 13 & 0.0049697 & 0.96592 \\
\hline 14 & 0.00470773 & 0.9804 & 14 & 0.0040182 & 0.78098 \\
\hline 15 & 0.00405562 & 0.84459 & 15 & 0.00361484 & 0.70258 \\
\hline 16 & 0.00359747 & 0.74918 & 16 & 0.0028407 & 0.55212 \\
\hline 17 & 0.00257517 & 0.53629 & 17 & 0.00226606 & 0.44043 \\
\hline 18 & 0.00202225 & 0.42114 & 18 & 0.00166779 & 0.32415 \\
\hline 19 & 0.00162911 & 0.33927 & 19 & 0.00124976 & 0.2429 \\
\hline 20 & 0.000892907 & 0.18595 & 20 & 0.000902785 & 0.17547 \\
\hline 21 & $3.01 \mathrm{E}-16$ & $6.27 \mathrm{E}-14$ & 21 & 2.74E-16 & $5.34 \mathrm{E}-14$ \\
\hline
\end{tabular}

Cuadro 4. Vectores propios, obtenidos del análisis de componentes principales (ACP) para los Bryconamericus del Pacífico de Colombia, Ecuador y Perú. CP: componente principal

\begin{tabular}{|c|c|c|c|}
\hline \multirow[b]{2}{*}{ Caracteres } & \multicolumn{3}{|c|}{ Vectores propios } \\
\hline & $C P 1$ & $C P 2$ & CP3 \\
\hline Longitud estándard & -0.02268 & 0.003772 & -0.07375 \\
\hline Longitud total & -0.01769 & -0.03841 & -0.06512 \\
\hline Longitud hocico-aletas pélvicas & 0.003819 & -0.02284 & -0.1095 \\
\hline Longitud hocico-aleta anal & -0.01098 & -0.02 & -0.06185 \\
\hline Longitud hocico-aletas pectorales & 0.005834 & -0.1294 & -0.04031 \\
\hline Longitud hocico-aleta dorsal & -0.01588 & -0.02762 & -0.09102 \\
\hline Longitud aleta dorsal-aleta anal & -0.0355 & -0.1052 & 0.08646 \\
\hline Longitud aleta dorsal-aletas pectorales & -0.01069 & -0.03686 & -0.07056 \\
\hline Profundidad del pedúnculo caudal & -0.05126 & 0.178 & -0.03302 \\
\hline Longitud pedúnculo caudal & -0.2119 & 0.7504 & -0.1912 \\
\hline Longitud aletas pectorales & 0.003225 & -0.1146 & 0.05773 \\
\hline Longitud aletas pélvicas & 0.03426 & -0.03737 & 0.004824 \\
\hline Longitud aleta dorsal & -0.02037 & -0.1331 & 0.1708 \\
\hline Longitud aleta anal & 0.01755 & -0.1744 & 0.2092 \\
\hline Longitud de la cabeza & -0.07846 & -0.1324 & -0.01218 \\
\hline Longitud aleta dorsal-hipurales & -0.3099 & 0.1172 & 0.7157 \\
\hline Longitud postorbital de la cabeza & -0.2121 & 0.01407 & -0.09586 \\
\hline Longitud del hocico & 0.04727 & -0.08709 & -0.345 \\
\hline Diámetro del ojo & 0.08326 & -0.3811 & 0.1232 \\
\hline Longitud hueso maxilar & -0.05052 & -0.257 & -0.3963 \\
\hline Longitud mandíbula superior & 0.8883 & 0.2447 & 0.1596 \\
\hline
\end{tabular}




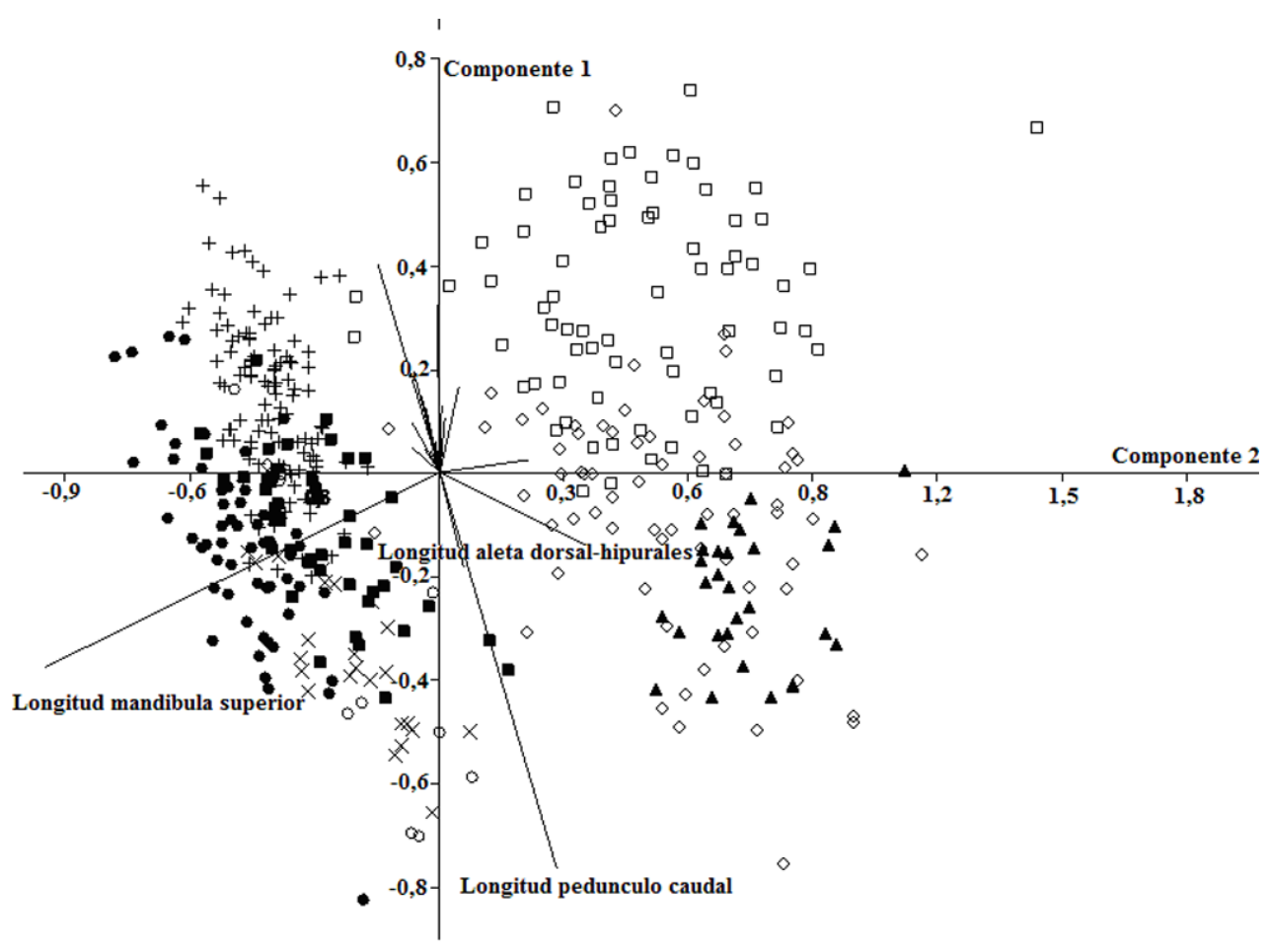

Figura 2. Análisis de componentes principales (ACP) de la variación morfológica de las especies de Bryconamericus: B. brevirostris $(+)$, B. dahli ( $\square)$, B. guaytarae (x), B. miraensis (०), B. peruanus $(\bullet)$, Bryconamericus sp. $1(\diamond)$, Bryconamericus sp. $2(\mathbf{\Delta})$ y Bryconamericus sp. 3 (匹).

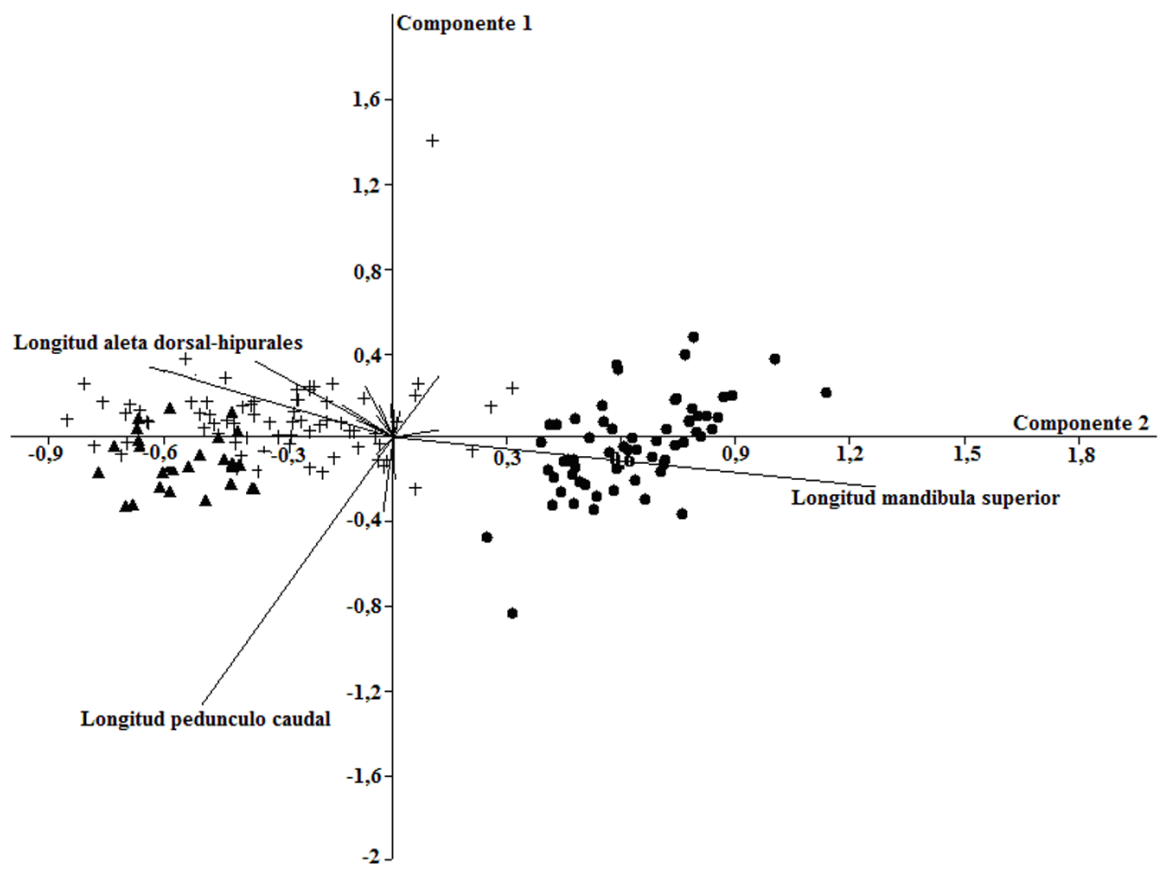

Figura 3. Análisis de componentes principales (ACP) de la variación morfológica de lo registrado como B. peruanus: B. peruanus $(\bullet)$, Bryconamericus sp. 1 (+) y Bryconamericus sp. 2 ( $\mathbf{\Delta})$. 
embargo, el número de escamas con poros en la línea lateral permiten diferenciar a B. peruanus y Bryconamericus sp. 1 de Bryconamericus sp. 2 (Kruskal Wallis: $p=0.000$ ), y el número total de radios de la aleta anal separa $B$. peruanus de Bryconamericus sp. 3 (Kruskal Wallis: $p<0.0001)$ ).

Por lo tanto, se identificaron 3 especies de Bryconamericus para el Pacífico de Ecuador: B. dahli, B. brevirostris y Bryconamericus sp.1; para el Amazonas de Ecuador, 1 especie: Bryconamericus sp. 2; para el Pacífico sur de Colombia 4 especies: B. miraensis, B. dahli, B. guaytarae y Bryconamericus sp. 3; para la vertiente del Pacífico de Perú, 1 especie: $B$. peruanus (Figs. 4 y 5). Se encontró que la cuenca hidrográfica del Pacífico de Colombia y Ecuador comparten la distribución de B. dahli (Fig. 4). Bryconamericus peruanus se restringe al sistema hidrográfico del Pacíifico en Perú (Fig. 5) y está en simpatría con B. brevirostris en el río Tumbes.

\section{Discusión}

A partir de la variación en morfometría, merística y la presencia de ganchos en todas las aletas con radios, la distribución de $B$. peruanus se restringe al sistema hidrográfico del Pacífico en Perú (Fig. 5). Lo registrado como
B. peruanus para el Pacífico de Ecuador (Román-Valencia, 2003a; b; 2005) coincide con la presencia de ganchos en todas las aletas con radios de Bryconamericus sp.1: no obstante, y de acuerdo al análisis de componentes principales, difiere completamente de la forma típica de B. peruanus (véase Resultados). Aquí se considera una especie de Bryconamericus por describir (=Bryconamericus sp.1). Böhlke (1958) registra B. peruanus para el alto Amazonas de Ecuador. Este registro se diferencia del material de B. peruanus aquí examinado por la presencia de ganchos sólo en los radios de las aletas anal y pélvica; por lo que se considera un registro erróneo de Böhlke (1958) y una especie de Bryconamericus por describir. Anotan Wiley y Collette (1970) que los machos de $B$. peruanus presentan ganchos en todas las aletas sostenidas por radios (excepto en la aleta caudal), lo que coincide con este trabajo y permite identificar $B$. peruanus para el Pacífico de Perú. Los registros para el Pacífico y Amazonas de Ecuador y el Pacífico sur de Colombia se reconocen aquí como 3 especies de Bryconamericus por describir.

El uso de caracteres como número de vértebras, radios procurrentes y pterigióforos no permitió diferenciar las especies de Bryconamericus del Pacífico de Colombia y Ecuador, lo que concuerda con las observaciones de
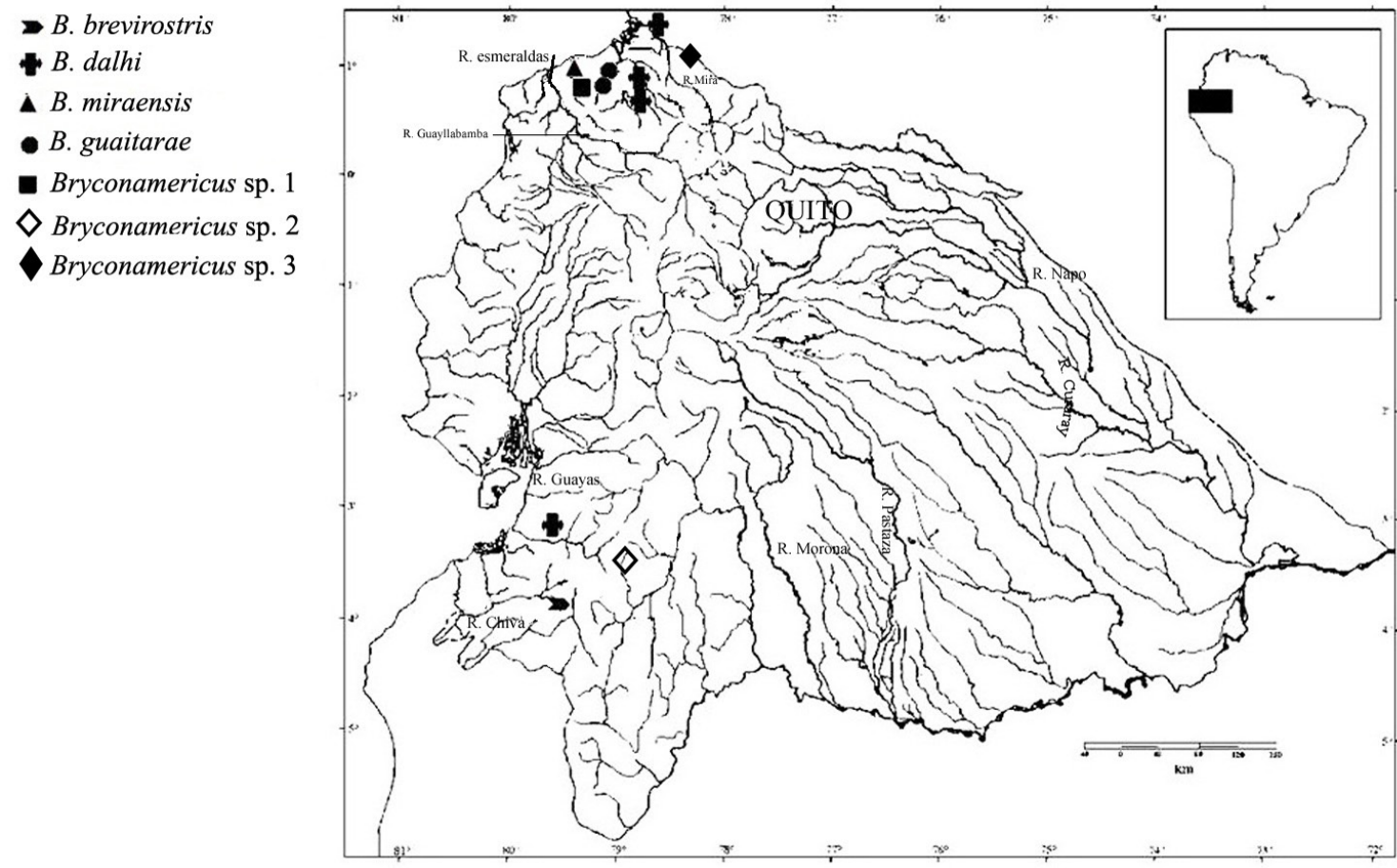

Figura 4. Modelo de distribución de las especies de Bryconamericus del Pacífico de Colombia y Ecuador. 


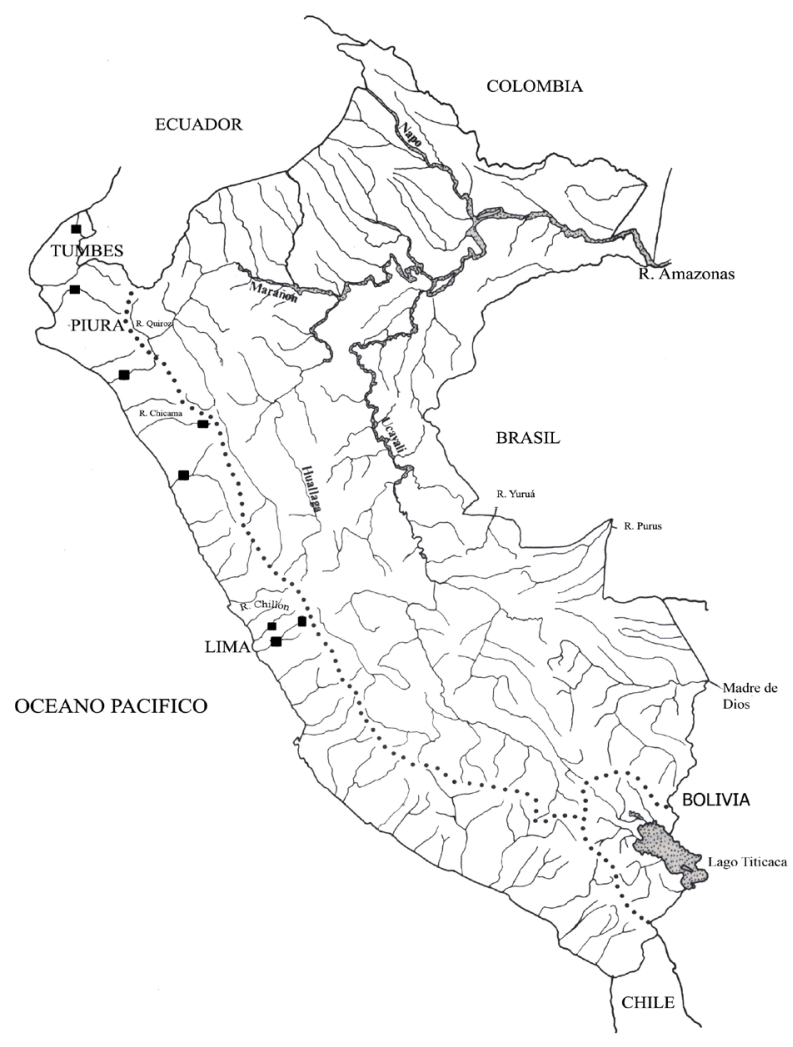

Figura 5. Distribución geográfica de Bryconamericus peruanus (a).

Román-Valencia et al. (2009a; b) para especies de Bryconamericus y de Hemibrycon, respectivamente; no obstante, los ganchos en las aletas en el caso de los machos, el número de escamas con poros en la línea lateral, las escamas predorsales y el número total de radios en la aleta anal fueron útiles en la diferenciación de $B$. brevirostris, $B$. miraensis, B. peruanus, B. dahli, B. guaytarae, Bryconamericus sp. 1, Bryconamericus sp. 2 y Bryconamericus sp. 3 , situación parecida a la reportada por Román-Valencia et al. (2009 b; c).

Bryconamericus sp. 2 y Bryconamericus sp. 3 se separan de B. peruanus y Bryconamericus sp. 1, por la presencia de ganchos sólo en los radios de las aletas anal y pélvica ( $v s$. ganchos en todas las aletas con radios, excepto la aleta caudal). Los caracteres merísticos, como número de escamas con poros en la línea lateral y número de radios totales en la aleta anal permitieron distinguir a Bryconamericus sp. 1 y Bryconamericus sp. 2 de B. brevirostris, $B$. miraensis, $B$. dahli, B. guaytarae.

La distribución de las especies de Bryconamericus en el Pacífico de Colombia, Ecuador y Perú, la similitud morfológica y diferencias asociadas a caracteres merísti- cos y del dimorfismo sexual, evidencian la diversificación de Bryconamericus en Suramérica y la influencia del aislamiento de las vertientes hidrográficas del Pacífico de Colombia, Ecuador y Perú en los procesos de especiación de este grupo, consecuencia de los eventos geológicos de la cordillera de los Andes (Román-Valencia y Vanegas Rios, 2009; Román-Valencia et al., 2009b). También las especies de Bryconamericus del bajo América Central formaron un grupo monofilètico y se encontraron relacionados con otras especies del Pacifico de América del Sur (Román-Valencia y Vanegas Ríos, 2009).

\section{Agradecimientos}

Se recibió financiación de la Universidad del QuindíoVicerrectoria de Investigaciones (proyectos 212, 304 y 462) e Idea Wild. Agradecemos al Grupo de Investigación y Asesoría en Estadística de la Universidad del Quindío, en especial a H. Hurtado y D. Galvis por sus comentarios, discusión y sugerencias, y a Peter Bartsch (ZMB) por el generoso envío de fotografías de los sintipos de B. peruanus (figura 1). Las siguientes personas prestaron o donaron material de comparación: Mark H. Sabaj P. (ANSP), James Maclaine (BMNH), John Fong, David Catania y Mysi Hoang (CAS), Janeth Muñoz y Jaime Aguirre C. (ICNMNH), Ramiro Barriga (MEPN), Richard P. Vari y Susan L. Jewett (USNM). Dos revisores anónimos leyeron versiones del artículo y plantearon valiosas correcciones, críticas y sugerencias. Carlos A. Garcia-A. (IUQ) elaboró las figuras 4 y 5. Donald C. Taphorn leyó el resumen en inglés.

\section{Literatura citada}

Böhlke, E. J. 1958. Studies of the family Characidae. A report on several extensive recent collections from Ecuador. Proceedings of the Academy of Natural Sciences of Philadelphia 110:1-121.

Burnaby, T. P. 1966. Growth-invariant discriminant functions and generalized distances, Biometrics 22:96-110.

Eigenmann, C. H. 1927. The American Characidae. Memoirs of the Museum of Comparative Zoology 43:311-428.

Fink, W. L. y S. H. Weitzman. 1974. The so-called Cheirodontin fishes of Central America with descriptions of two new species (Pisces: Characidae). Smithsonian Contributions to Zoology 172:1-46.

Hammer, Ø., D A. T. Harper y P. D. Ryan. 2001. PAST: Paleontological statistics software package for education and data analysis. Paleontología Electrónica 4:9.

Müller, J. y F. H. Troschel. 1845. Horae Ichthyologicae. Beschreibung und Abbildung neuer Fische. Die Familie der Characinen, Erstes und Zweites Heft. Berlin 40 p. 
Ortega, H. y R. P. Vari. 1986. Annotated checklist of the freshwater fishes of Peru. Smithsonian Contributions of Zoology 437:1-22.

Román-Valencia, C. 2002. Revisión sistemática de las especies del género Bryconamericus (Teleostei: Characidae) de Centroamérica. International Journal of Tropical Biology and Conservation 50:173-192.

Román-Valencia, C. 2003a. Description of a new species of Bryconamericus (Teleostei: Characidae) from the Amazon. Bollettino del Museo Regionale di Scienze Naturali di Torino 20:477-486.

Román-Valencia, C. 2003b. Sistemática de las especies colombianas de Bryconamericus (Characiformes, Characidae). Dahlia (Revista de la Asociación Colombiana de Ictiólogos) 6:17-58.

Román-Valencia, C. 2005. Sinopsis comentada de las especies del género Bryconamericus (Teleostei: Characidae) de Venezuela y norte del Ecuador, con la descripción de una nueva especie para Venezuela. Memorias de la Fundación La Salle de Ciencias Naturales, Caracas 163:27-52.

Román-Valencia, C., D. C. Taphorn y R. I. Ruiz. 2008a. Two new Bryconamericus: B. cinarucoense n.sp. and B. singularis n.sp. (Characiformes, Characidae) from the Cinaruco River, Orinoco Basin, with key to all Venezuelan Species. Animal Biodiversity and Conservation 31:15-27.

Román-Valencia, C., J. A. Vanegas-Ríos y R. I. Ruiz. 2008 b. Una nueva especie de pez del género Bryconamericus (Ostariophysi: Characidae) del río Magdalena, con una clave para las especies de Colombia. International Journal of Tropical Biology and Conservation 56:1749-1763.

Román-Valencia, C. y J. A. Vanegas-Ríos. 2009. Análisis filogenético y biogeográfico de las especies del género Bryconamericus (Characiformes, Characidae) de la baja América Central. Caldasia 32:393-406.

Román-Valencia, C., D. K. Arcila-Mesa y H. Hurtado. 2009a. Variación morfológica de los peces Hemibrycon boquiae y Hemibrycon rafaelense (Characiformes: Characidae) en el río Cauca, Colombia. International Journal of Tropical Biology and Conservation 57:541-556.

Román-Valencia, C., J. A. Vanegas-Ríos y M. D. García. 2009b.
Análisis comparado de las especies del género Bryconamericus (Teleostei: Characidae) en la cuenca de los ríos CaucaMagdalena y Ranchería, Colombia. Revista Mexicana de Biodiversidad 80:465-482.

Román-Valencia, C., D. K. Arcila-Mesa y M. D. García. 2009c. Diversidad fenotípica en peces del género Hemibrycon (Characiformes: Characidae) del sistema del río MagdalenaCauca, Colombia. Brenesia 71-72:27-40.

Román-Valencia, C., J. A. Vanegas-Ríos y R. I. Ruiz. 2009d. Especie nueva del género Bryconamericus (Teleostei: Characidae) del río Fonce, sistema río Magdalena, Colombia. Revista Mexicana de Biodiversidad 80:455-463.

Román-Valencia, C., C. García-Alzate, R. I. Ruiz y D. C. Taphorn. 2010. Bryconamericus macarenae n. sp. (Characiformes, Characidae) from the Güejar River, Macarena mountain range, Colombia. Animal Biodiversity and Conservation 32:195-203.

Sabaj-Perez, N. H. (editor). 2010. Standard symbolic codes institutions resource collections in Herpetology and Ichthyology: an on line reference, version 1.5. American Society Ichthyologist and Herpetologist, Washington, D.C. http.//www.asih.org/; última consulta: 4.X.2010.

Song, J. y L. R. Parenti. 1995. Clearing and staining whole fish specimens for simultaneous demonstration of bone, cartilage and nerves. Copeia 1995:114-118.

Statgraphics. 2005. Statgraphics centurion XV. User manual. Statpoint Technologies, Warrenton, Virginia. 283 p.

Taylor, W. R. y G. C. Van Dyke. 1985. Revised procedures for staining and clearing small fishes and other vertebrates for bone and cartilage study. Cybium 9:107-119.

Tortonese, E. 1941-1942. Ricerche ed osservazioni sui caracidi delle sottofamiglie Tetragonopterinae, Glandulocaudinae e Stethaprioninae (Teleostei Plectospondyli). Bollettino dei Musei di Zoologia ed Anatomia Comparata della R. Universita di Torino (Ser. 4) 49:11-86.

Wiley, M. L. y B. B. Collette. 1970. Breeding tubercles and contact organs in fishes: their occurrence, structure and significance. Bulletin of the American Museum of Natural History 143:145-216. 